\title{
Informational Steady States and Conditional Entropy Production in Continuously Monitored Systems
}

\author{
Gabriel T. Landi® , $^{1,{ }^{*}}$ Mauro Paternostro, ${ }^{2}$ and Alessio Belenchia $\odot^{3,2}$ \\ ${ }^{1}$ Instituto de Física da Universidade de São Paulo, São Paulo 05314-970, Brazil \\ ${ }^{2}$ Centre for Theoretical Atomic, Molecular, and Optical Physics, School of Mathematics and Physics, \\ Queens University, Belfast BT7 1NN, United Kingdom \\ ${ }^{3}$ Institut für Theoretische Physik, Eberhard-Karls-Universität Tübingen, Tübingen 72076, Germany
}

(Received 25 March 2021; accepted 10 December 2021; published 5 January 2022)

\begin{abstract}
We put forth a unifying formalism for the description of the thermodynamics of continuously monitored systems, where measurements are only performed on the environment connected to a system. We show, in particular, that the conditional and unconditional entropy production, which quantify the degree of irreversibility of the open system's dynamics, are related to each other by the Holevo quantity. This, in turn, can be further split into an information gain rate and loss rate, which provide conditions for the existence of informational steady states, i.e., stationary states of a conditional dynamics that are maintained owing to the unbroken acquisition of information. We illustrate the applicability of our framework through several examples.
\end{abstract}

DOI: 10.1103/PRXQuantum.3.010303

\section{INTRODUCTION}

The dynamics of a quantum system depends not only on itself, but also on how it is probed, showcasing the remarkable extrinsic character of quantum mechanics. This unavoidable backaction due to measurements can be directly probed in the laboratory [1-4], and is by far the most intriguing and dramatic aspect of quantum theory. It also has a clear thermodynamic flavor [5], since backaction is an intrinsically irreversible process. A comprehensive theory describing the thermodynamics of monitored systems would therefore greatly benefit our understanding of the interplay between information and dissipation. Constructing such a theory, however, is not trivial, since it requires reformulating the second law to take into account the information learned from the measurements. We call this a conditional second law. It quantifies which processes are allowed, given a certain set of measurement outcomes. Interestingly, due to measurement backaction, the noise introduced by the measurement can actually make the conditional process more irreversible, as recently demonstrated in a superconducting qubit experiment [6].

\footnotetext{
*gtlandi@if.usp.br
}

Published by the American Physical Society under the terms of the Creative Commons Attribution 4.0 International license. Further distribution of this work must maintain attribution to the author(s) and the published article's title, journal citation, and DOI.
When a system is coupled to two baths at different temperatures, it usually tends to a nonequilibrium steady state (NESS), where the competition between the two baths keeps the system away from equilibrium. Continuous measurements can lead to a similar effect. In this case, noise is constantly being introduced by the environment or the measurement backaction. But information is also constantly being acquired. These two effects compete, leading the system toward an informational steady state (ISS). Crucially, the ISS relies on the experimenter's knowledge of the measurement records. A beautiful experimental illustration of this effect was recently given in Ref. [7], where the authors studied an optomechanical membrane monitored by an optical field. By measuring the field, one could monitor the position of the mechanical membrane and thus infer a steady state that was close to the ground state. Conversely, if the measurements are not read, the membrane is perceived to be in a thermal state with higher temperatures. The ISS is therefore colder, due to the information acquired from the continuous measurement.

ISSs are just one example of the many interesting phenomena that emerge when quantum measurements are introduced in a thermodynamic picture. The deep connections between the two concepts, together with recent experimental advances in controlled quantum platforms, have led to a surge of interest in formulating conditional laws of thermodynamics [8-20]. This also motivated ground-breaking experiments applying these ideas to Maxwell demon engines and feedback control [6,21-24]. In all these frameworks, however, the 
measurements are assumed to act directly on the system, making them explicitly invasive.

Conversely, our interest in this paper will be on formulating the laws of thermodynamics when the measurements are done only on the environment and only after it interacted with the system. The scenario is therefore noninvasive by construction, so that any information acquired can only reduce the entropy produced in the process even if the measurement is very poor (as is often the case when dealing with large environments). This represents a change in philosophy compared to, e.g., Ref. [12], where the measurement was introduced by coupling the system to a memory and then measuring the memory. In that case one constructs the conditional second law by comparing the situation where the system is fully isolated with that in which it is open due to the interaction with the memory. In our case, we assume instead that the interaction between system and bath is inevitable and will happen whether or not we measure it. We then ask how measuring the bath affects the degree of irreversibility of the process.

Crucially, the framework we develop will focus on a continuously monitored system, in contrast to, e.g., Ref [12]. It is therefore particularly suited for describing ISSs. Our endeavor began in Ref. [20], where we put forth a semiclassical theory valid for Gaussian processes. We were interested in quantum optical experiments, which have already been using some of these ideas for many decades, in the framework of continuously monitored systems $[25,26]$. In fact, our theory was recently employed in Ref. [27] to experimentally assess the conditional second law in an optomechanical system. However, in addition to being semiclassical, the framework of Ref. [20] also has another serious limitation: it is formulated solely in terms of the stochastic master equation obeyed by the system; that is, it does not require an explicit model of the environment, but only which type of open dynamics it produces.

There has been increasing evidence that a proper formulation of thermodynamics in the quantum regime is only possible if information on the environment and the system-environment interactions are provided [28]. Reduced descriptions, based only on master equations, can show apparent violations of the second law [29], something that can only be resolved by introducing a specific model of the environment [30].

In this paper we put forth a very general framework for describing the thermodynamics of continuously monitored systems, where measurements are only done indirectly in the bath. The formalism applies to a broad variety of systems and processes, and is particularly suited for describing ISSs. The building block we use is to replace the continuous dynamics by a stroboscopic evolution in small time steps, described in terms of a collisional model (CM) [31-40]. This has two main advantages. First, the thermodynamics of CMs is by now very well understood
[30,40-43] (see also Ref. [28] for a recent review). And second, CMs naturally emerge in quantum optics, from a discretization of the field operator into discrete time bins $[44,45]$. The typical scenario is a system interacting with an optical cavity, where a constant flow of photons is injected by an external pump [cf. Fig. 1(a)]. At each time step, the system will only interact with a certain time window of the field, thus transforming the dynamics into that of a series of sequential collisions between the system and some ancilla. Because of this connection, collisional models serve as a convenient tool for constructing the framework of continuous measurements in experimentally relevant systems. We refer to these as continuously monitored collisional models $\left(\mathrm{CM}^{2} \mathrm{~s}\right)$.

Our paper is organized as follows. Section II establishes the basic framework, including the collisional setup. The corresponding information flows and thermodynamic features are characterized in Sec. III, which also contains the main contribution of this work: namely the construction of a conditional second law, which is capable of capturing the interplay between thermodynamics and information. In Sec. IV, we apply the $\mathrm{CM}^{2}$ framework to models involving qubits providing some illustrative applications. Accompanying this manuscript, we also make publicly available a self-contained numerical library in MATHEMATICA for carrying out stochastic simulations of $\mathrm{CM}^{2} \mathrm{~s}$ [46]. Finally, in Sec. V we draw our conclusions and highlight the perspectives opened by our approach.

\section{CONTINUOUSLY MEASURED COLLISIONAL MODELS}

Here we develop the basic framework of a $\mathrm{CM}^{2}$. We consider a system $X$, with initial density matrix $\rho_{X_{0}}$, which is put to interact sequentially with a series of independent and identically prepared ancillae, labeled $Y_{1}, Y_{2}$, etc., and prepared always in the same state $\rho_{Y_{t}}=\rho_{Y}$. Time is labeled in discrete units of $t=0,1,2,3, \ldots$. The collision taking the system from $t-1$ to $t$ is described by a unitary $U_{t}$ [47] acting only between the system $X$ and ancilla $Y_{t}$ as [Fig. 1(b)]

$$
\rho_{X_{t} Y_{t}^{\prime}}=U_{t}\left(\rho_{X_{t-1}} \otimes \rho_{Y_{t}}\right) U_{t}^{\dagger},
$$

where $Y_{t}^{\prime}$ refers to the state of ancilla $Y_{t}$ after the collision. Taking the partial trace over the ancilla leads to the stroboscopic (Markovian) map

$$
\rho_{X_{t}}=\mathcal{E}\left(\rho_{X_{t-1}}\right):=\operatorname{tr}_{Y_{t}}\left\{\rho_{X_{t} Y_{t}^{\prime}}\right\}
$$

Note that $\mathcal{E}$ does not need to carry an index $t$, since it is the same for all collisions. After such a map, the ancilla $Y_{t}^{\prime}$ never participates again in the dynamics and, for the next step, a fresh ancilla $Y_{t+1}$ is introduced and the map in Eq. (2) is repeated. 
(a)

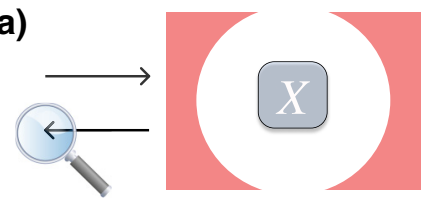

(b)

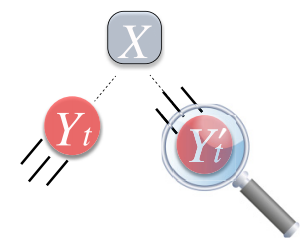

(c)

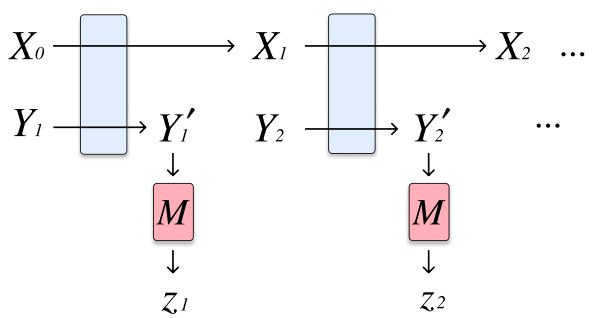

(d)

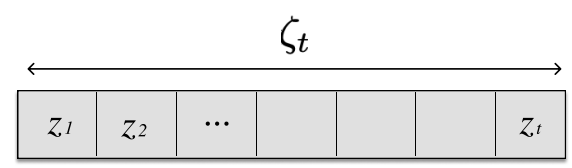

FIG. 1. (a) A typical method for continuously monitoring a system is to couple it to an optical cavity and measure the photons leaking out. (b) In a collisional model picture, the monitoring is introduced instead through a series of sequential collisions between the system $X$ and independent ancillae $Y_{t}$, which are subjected to measurement after each collision. (c) Diagrammatic representation of the model. The system is described stroboscopically (discrete time) by a state $\rho_{X_{t}}$. At each instant of time, it interacts with an independent ancilla, prepared in state $\rho_{Y}$, according to the map in Eq. (1). Afterwards, the ancillae are measured, as described by generalized measurement operators $\left\{M_{z}\right\}$, which produce a classical (and random) outcome $z_{t}$. (d) As time progresses, one builds up a measurement record $\zeta_{t}=\left(z_{1}, \ldots, z_{t}\right)$, which contains all the information acquired about $X$ up to time $t$.

Information on the state of the system is acquired indirectly by measuring the states $\rho_{Y_{t}^{\prime}}$ of each ancilla after they collided with $X$. The measurement is described by a set of generalized measurement operators $\left\{M_{z}\right\}$, satisfying $\sum_{z} M_{z}^{\dagger} M_{z}=\mathbb{1}$, so that outcome $z_{t}$ occurs with probability

$$
P\left(z_{t}\right)=\operatorname{tr}\left\{M_{z_{t}} \rho_{Y_{t}^{\prime}} M_{z_{t}}^{\dagger}\right\}
$$

By using generalized measurements, we encompass both projective, as well as weak measurements in the bath. A diagrammatic depiction of the dynamics is shown in Fig. 1(c). A $\mathrm{CM}^{2}$ is completely described by specifying $\left\{\rho_{Y}, U, M_{z}\right\}$.

The distribution in Eq. (3) concerns only the marginal statistics of a single outcome. Our interest will be instead on the joint statistics of the set of measurement records

$$
\zeta_{t}=\left(z_{1}, \ldots, z_{t}\right)
$$

The indices are chosen so that $\zeta_{t}$ contains all information about the system available up to time $t$. As $\zeta$ encompasses the entire measurement record, it is associated with the "integrated" information on $X$. Conversely, $z_{t}$ represents a differential information gain associated only with the step $X_{t-1} \rightarrow X_{t}$ [Fig. 1(d)]. The joint distribution $P\left(\zeta_{t}\right)$ is given by

$$
P\left(\zeta_{t}\right)=\operatorname{tr}_{X Y_{1} \cdots Y_{t}}\left\{M_{z_{t}} \cdots M_{z_{1}} \rho_{X Y_{1} \cdots Y_{t}} M_{z_{1}}^{\dagger} \cdots M_{z_{t}}^{\dagger}\right\},
$$

where

$$
\rho_{X Y_{1} \cdots Y_{t}}=\left(\prod_{k=1}^{t} U_{k}\right)\left(\rho_{X_{0}} \bigotimes_{j=1}^{t} \rho_{Y_{j}}\right)\left(\prod_{k=1}^{t} U_{k}\right)^{\dagger} .
$$

The factorization in this last expression stems from the fact that $U_{t}$ only has support between $X$ and $Y_{t}$, and hence commutes with all other ancilla states $\rho_{Y_{t}^{\prime}}, t^{\prime} \neq t$. Note also that, since the measurements act only on those ancillae that no longer participate in the dynamics, it is irrelevant whether the measurement $M_{z_{t}}$ occurs before the next evolution with $Y_{t+1}$ or not.

Finally, we also require the conditional state of the system $\rho_{X_{t} \mid \zeta_{t}}$, which quantifies the knowledge the experimenter has about the system, given that the measurement record $\zeta_{t}$ is observed. Such a state is given by

$$
\rho_{X_{t} \mid \zeta_{t}}=\frac{1}{P\left(\zeta_{t}\right)} \operatorname{tr}_{Y_{1} \ldots Y_{t}}\left\{\left(\prod_{k=1}^{t} M_{z_{k}}\right) \rho_{X Y_{1} \cdots Y_{t}}\left(\prod_{k=1}^{t} M_{z_{k}}\right)^{\dagger}\right\}
$$

As the measurements are performed only on the ancillae, there is never a direct backaction on the system, which is expressed mathematically by

$$
\sum_{\zeta_{t}} P\left(\zeta_{t}\right) \rho_{X_{t} \mid \zeta_{t}}=\rho_{X_{t}}
$$

for any choice of generalized measurements $\left\{M_{z}\right\}$. That is, the average of $\rho_{X_{t} \mid \zeta_{t}}$ over all outcomes $\zeta_{t}$ yields back the unconditional state $\rho_{X_{t}}$. Thus, while there may be a conditional backaction, unconditionally the measurement is noninvasive.

The normalization factor $P\left(\zeta_{t}\right)$ in Eq. (6) introduces an unwanted complication, as it forbids us to write $\rho_{X_{t} \mid \zeta_{t}}$ as a map acting on $\rho_{X_{t-1} \mid \zeta_{t-1}}$. This can be resolved, however, if we work with unnormalized states. We define the 
completely positive, trace nonpreserving map

$$
\mathcal{E}_{z}\left(\rho_{X}\right)=\operatorname{tr}_{Y}\left\{M_{z} U\left(\rho_{X} \otimes \rho_{Y}\right) U^{\dagger} M_{z}^{\dagger}\right\},
$$

which is indexed by the possible outcomes $z$ of the measurements. Instead of working with $\rho_{X_{t} \mid \zeta_{t}}$ in Eq. (6), we consider the unnormalized states $\varrho_{X_{t} \mid \zeta_{t}}$, defined as the sequence generated by the map $\varrho_{X_{t} \mid \zeta_{t}}=\mathcal{E}_{z_{t}}\left(\varrho_{X_{t-1} \mid \zeta_{t-1}}\right)$ with initial condition $\varrho_{X_{0} \mid \zeta_{0}}=\rho_{X_{0}}$. One may readily verify that

$$
\operatorname{tr}_{X} \varrho_{X_{t} \mid \zeta_{t}}=\operatorname{tr}_{X}\left\{\mathcal{E}_{z_{t}} \circ \cdots \circ \mathcal{E}_{z_{1}}\left(\rho_{X_{0}}\right)\right\}=P\left(\zeta_{t}\right) .
$$

The states $\varrho_{X_{t} \mid \zeta_{t}}$ therefore contain the outcome distribution $P\left(\zeta_{t}\right)$ at any given time. And the normalized state in Eq. (6) is recovered as $\rho_{X_{t} \mid \zeta_{t}}=\varrho_{X_{t} \mid \zeta_{t}} / P\left(\zeta_{t}\right)$.

It is useful to keep in mind the interpretation of a $\mathrm{CM}^{2}$ as a hidden Markov model $[9,48,49]$. The system evolution is Markovian, but this is hidden from the observer who is partially ignorant about its dynamics: access to $X$ is only possible through the classical outcomes $\zeta_{t}$. In the language of Bayesian networks, the key issue entailed by our framework is thus about the predictions that can be made on the state of the hidden layer $X$ given the information available through the visible layer of the outcomes $\zeta_{t}$ only. This highlights the nice interplay between quantum and classical features present in these models: The evolution of the system is quantum, but information is only accessed through classical data. We have also found it illuminating to understand what would be the classical version of a $\mathrm{CM}^{2}$, as this allows us to relate our framework directly with the classical formalism of Ito, Sagawa, and Ueda $[9,10]$. This is addressed in Appendix A, where we also discuss the conditions for a $\mathrm{CM}^{2}$ to be incoherent.

\section{INFORMATION AND THERMODYNAMICS}

\section{A. Quantum-classical information}

The information content in the unconditional state $\rho_{X_{t}}$ can be quantified by the von Neumann entropy $S\left(X_{t}\right) \equiv$ $S\left(\rho_{X_{t}}\right)=-\operatorname{tr} \rho_{X_{t}} \ln \rho_{X_{t}}$. Similarly, the information in the conditional state $\rho_{X_{t} \mid \zeta_{t}}$ (properly normalized) is quantified by the quantum-classical conditional entropy

$$
S\left(X_{t} \mid \zeta_{t}\right)=\sum_{\zeta_{t}} P\left(\zeta_{t}\right) S\left(\rho_{X_{t} \mid \zeta_{t}}\right)
$$

Each term $S\left(\rho_{X_{t} \mid \zeta_{t}}\right)$ quantifies the information for one specific realization $\zeta_{t}$, and $S\left(X_{t} \mid \zeta_{t}\right)$ is then an average over all trajectories. Equation (10) is already averaging over $\zeta_{t}$, even though $\zeta_{t}$ is referenced explicitly on the left-hand side. Albeit possibly confusing at first, this is a standard notation in information theory. Note also that Eq. (10) is not the quantum conditional entropy, a quantity which can be negative. Here, since we are conditioning on classical outcomes, $S\left(X_{t} \mid \zeta_{t}\right)$ is always strictly non-negative. In this paper all conditional entropies will be of this form.
The mismatch between $S\left(X_{t}\right)$ and $S\left(X_{t} \mid \zeta_{t}\right)$ is given by the Holevo information (or Holevo quantity) [50]

$$
I\left(X_{t}: \zeta_{t}\right):=S\left(X_{t}\right)-S\left(X_{t} \mid \zeta_{t}\right)
$$

It quantifies the information about $X$ contained in the classical outcomes $\zeta_{t}$. Its interpretation becomes clearer by casting it as

$$
I\left(X_{t}: \zeta_{t}\right)=\sum_{\zeta_{t}} P\left(\zeta_{t}\right) D\left(\rho_{X_{t} \mid \zeta_{t}}|| \rho_{X_{t}}\right) \geq 0
$$

where $D(\rho \| \sigma)=\operatorname{tr}(\rho \ln \rho-\rho \ln \sigma)$ is the quantum relative entropy. Therefore, $I\left(X_{t}: \zeta_{t}\right)$ is the weighted average of the "distance" between $\rho_{X_{t} \mid \zeta_{t}}$ and $\rho_{X_{t}}$.

The Holevo information reflects the integrated information, acquired about the system, up to time $t$. This is different from the small increment that is obtained from a single outcome $z$, at each step. In order to quantify such differential information gain, the natural quantity is the conditional Holevo information

$$
\begin{aligned}
G_{t}:=I_{c}\left(X_{t}: z_{t} \mid \zeta_{t-1}\right) & =I\left(X_{t}: \zeta_{t}\right)-I\left(X_{t}: \zeta_{t-1}\right) \\
& =S\left(X_{t} \mid \zeta_{t-1}\right)-S\left(X_{t} \mid \zeta_{t}\right)
\end{aligned}
$$

It describes the correlations between $X_{t}$ and the latest available outcome $z_{t}$, given the past outcomes $\zeta_{t-1}=$ $\left(z_{1}, \ldots, z_{t-1}\right)$. The first term involves the state $\rho_{X_{t} \mid \zeta_{t-1}}$, which stands for the state of the system at time $t$, conditioned on all measurement records, except the last one. In symbols, it can thus be written as

$$
\rho_{X_{t} \mid \zeta_{t-1}}=\mathcal{E}\left(\rho_{X_{t-1} \mid \zeta_{t-1}}\right)
$$

where $\mathcal{E}$ is the unconditional map in Eq. (2). This therefore affords a beautiful interpretation to Eq. (13). Starting at $\rho_{X_{t-1} \mid \zeta_{t-1}}$, one compares two paths: a conditional evolution taking $\rho_{X_{t-1} \mid \zeta_{t-1}} \rightarrow \rho_{X_{t} \mid \zeta_{t}}$ and a unconditional evolution taking $\rho_{X_{t-1} \mid \zeta_{t-1}} \rightarrow \rho_{X_{t} \mid \zeta_{t-1}}$. Equation (13) measures the gain in information of the latter, compared to the former.

\section{B. Information rates and informational steady states}

Equation (11) is always non-negative. However, this does not imply that it will necessarily increase with time. In fact, the information rate

$$
\Delta I_{t}:=I\left(X_{t}: \zeta_{t}\right)-I\left(X_{t-1}: \zeta_{t-1}\right)
$$

can take any sign. This reflects the trade-off between the gain in information and the measurement backaction. A natural question is then whether it is possible to split $\Delta I_{t}$ as the difference between two strictly non-negative terms, the 
first naturally identified with the differential gain of information (13), and the second to the differential information loss. That is, whether a splitting of the form

$$
\Delta I_{t}=G_{t}-L_{t}
$$

would lead to the identification of a loss term $L_{t}$ that is strictly non-negative. As we will see in what follows, the answer to this question is in the positive.

To find a formula for $L_{t}$, we simply insert the first line of Eq. (13) into Eq. (15) to find that

$$
L_{t}:=I\left(X_{t-1}: \zeta_{t-1}\right)-I\left(X_{t}: \zeta_{t-1}\right)
$$

This is already clearly interpretable as a loss term, as it measures how information is degraded by the map in Eq. (14) and stems from the very collision with the ancillae invariably introducing extra noise in the system. Indeed, we can show that it is strictly non-negative. To do that, we use Eq. (12) to write $L_{t}$ as

$$
L_{t}=\sum_{\zeta_{t-1}} P\left(\zeta_{t-1}\right)\left[D\left(\rho_{X_{t-1} \mid \zeta_{t-1}} \| \rho_{X_{t-1}}\right)-D\left(\rho_{X_{t} \mid \zeta_{t-1}} \| \rho_{X_{t}}\right)\right] .
$$

But $\rho_{X_{t}}=\mathcal{E}\left(\rho_{X_{t-1}}\right)$ [Eq. (2)] and $\rho_{X_{t} \mid \zeta_{t-1}}=\mathcal{E}\left(\rho_{X_{t-1} \mid \zeta_{t-1}}\right)$ [Eq. (14)]. Together with the data processing inequality [51], this is enough to ascertain the non-negativity of $L_{t}$ for any quantum channel $\mathcal{E}$.

In the long-time limit the system may reach a steady state where $I_{\infty}$ no longer changes, so $\Delta I_{\infty}=0$. This does not necessarily mean that $G_{\infty}=L_{\infty}=0$, however. It might simply stem from a mutual balancing of gains and losses. That is, $G_{\infty}=L_{\infty} \neq 0$. We define an ISS as the asymptotic state for which

$$
\Delta I_{\mathrm{ISS}}=0 \quad \text { but } \quad G_{\mathrm{ISS}}=L_{\mathrm{ISS}} \neq 0 .
$$

In an ISS, information is continuously acquired, but this is balanced by the noise that is introduced by the measurement. Crucially, the ISS does not mean that $\rho_{X_{t} \mid \zeta_{t}}$ is no longer changing. This state is stochastic and thus continues to evolve indefinitely. Instead, what becomes stationary is the stochastic distribution of states in the state space [52].

Next we address the conditions for the existence of an ISS. First, one must bear in mind that the ISS depends on the choice of measurement, and it is always possible to choose a completely uninformative measurement $\left\{M_{z}\right\}$, making the problem pathological. This flexibility in the choice of measurement means that one can only provide necessary (and never sufficient) conditions for $G_{\text {ISS }} \neq 0$. As we now show, this condition boils down to whether or not the system and ancillae continue to correlate with each other, even in the long-time limit. More precisely, for a given history $\zeta_{t-1}$, consider the state

$$
\rho_{X_{t} Y_{t}^{\prime} \mid \zeta_{t-1}}=U_{t}\left(\rho_{X_{t-1} \mid \zeta_{t-1}} \otimes \rho_{Y_{t}}\right) U_{t}^{\dagger} .
$$

An ISS will not exist if, in the limit $t \rightarrow \infty$, the conditional state (20) factors into a product state for all $\zeta_{t-1}$ with nonzero support. This follows from the fact that, as discussed in Appendix B, the gain rate $G_{t}$ is bounded by

$$
G_{t} \leq \sum_{\zeta_{t-1}} P\left(\zeta_{t-1}\right) \mathcal{I}\left(\rho_{X_{t} Y_{t}^{\prime} \mid \zeta_{t-1}}\right)
$$

where $\mathcal{I}\left(\rho_{A B}\right)=S\left(\rho_{A}\right)+S\left(\rho_{B}\right)-S\left(\rho_{A B}\right)$ is the full quantum mutual information. Since $\mathcal{I}\left(\rho_{A B}\right)=0$ if and only if $\rho_{A B}=\rho_{A} \otimes \rho_{B}$, we are only guaranteed to have $G_{\text {ISS }} \equiv 0$ if all states (20) (with finite support) factor into a product. This analysis therefore shows that ISSs should in general be very easy to construct. In fact, similar considerations could be made concerning equilibrium versus nonequilibrium steady states.

\section{Unconditional second law}

Next we turn to the thermodynamics. The second law of thermodynamics characterizes the degree of irreversibility of a certain process and can be formulated in purely information-theoretic terms. This allows it to be extended beyond standard thermal environments, and also to avoid difficulties associated with the definitions of heat and work, which can be quite problematic in the quantum regime [28].

At each collision, the entropy of the system will change from $S\left(X_{t}\right)$ to $S\left(X_{t+1}\right)$. This change, however, may be either positive or negative. The goal of the second law is to identify a contribution to this change associated with the flow of entropy between the system and ancilla, and another representing the entropy that is irreversibly produced in the process. The separation thus takes the form

$$
\Delta \Sigma_{t}^{u}=S\left(X_{t}\right)-S\left(X_{t-1}\right)+\Delta \Phi_{t}^{u},
$$

where $\Delta \Phi_{t}^{u}$ is the unconditional flow rate of entropy from the system to the ancilla in each collision, and $\Delta \Sigma_{t}^{u}$ is the unconditional rate of entropy produced in the process. The second law is summarized by the statement that we should have $\Delta \Sigma_{t}^{u} \geq 0$. Equation (22) is merely a definition, however. The goal is precisely to determine the actual forms of $\Delta \Phi_{t}^{u}$ and $\Delta \Sigma_{t}^{u}$.

In standard thermal processes, this is usually accomplished by postulating that the entropy flow $\Delta \Phi_{t}^{u}$ should be linked with the heat flow $\dot{Q}_{t}$ entering the ancillae through Clausius' expression [53] $\Delta \Phi_{t}^{u}=\beta \dot{Q}_{t}$, where $\beta$ is the inverse temperature of the thermal state the ancillae are in. By fixing $\Delta \Phi_{t}^{u}$ we then also fix $\Delta \Sigma_{t}^{u}$. This, however, 
only holds for thermal ancillae, thus restricting the range of applicability of the formalism.

Instead, we approach the problem using the framework developed in Ref. [54] (see also Refs. [40,55]), which formulates the entropy production rate in informationtheoretic terms as

$$
\Delta \Sigma_{t}^{u}=\mathcal{I}\left(X_{t}: Y_{t}^{\prime}\right)+D\left(Y_{t}^{\prime} \| Y_{t}\right) \geq 0,
$$

where $\mathcal{I}\left(X_{t}: Y_{t}^{\prime}\right)=S\left(\rho_{X_{t}}\right)+S\left(\rho_{Y_{t}}^{\prime}\right)-S\left(\rho_{X_{t} Y_{t}^{\prime}}\right)$ is the quantum mutual information between the system and ancilla after Eq. (1) and $D\left(Y_{t}^{\prime} \| Y_{t}\right)=D\left(\rho_{Y_{t}^{\prime}} \| \rho_{Y_{t}}\right)$ is the relative entropy between the state of the ancilla before and after the collision. The first term thus accounts for the correlations that built up between the system and ancilla, while the second measures the amount by which the ancillae were pushed away from their initial states. Thus, from the perspective of the system, irreversibility stems from tracing over the ancillae after the interaction in such a way that all quantities related either to the local state of the ancilla, or to their global correlations, are irretrievable [55].

As the global map in Eq. (1) is unitary, and the system and ancillae are always uncorrelated before a collision, it follows that

$$
S\left(\rho_{X_{t} Y_{t}^{\prime}}\right)=S\left(\rho_{X_{t-1} Y_{t}}\right)=S\left(\rho_{X_{t-1}}\right)+S\left(\rho_{Y_{t}}\right) .
$$

Hence, the mutual information may also be written as

$$
\mathcal{I}\left(X_{t}: Y_{t}^{\prime}\right)=S\left(X_{t}\right)+S\left(Y_{t}^{\prime}\right)-S\left(X_{t-1}\right)-S\left(Y_{t}\right) .
$$

Substituting this into Eq. (23) and comparing with Eq. (22) then allows us to identify the entropy flux as

$$
\Delta \Phi_{t}^{u}=S\left(Y_{t}^{\prime}\right)-S\left(Y_{t}\right)+D\left(Y_{t}^{\prime} \| Y_{t}\right)=\operatorname{tr}\left\{\left(\rho_{Y_{t}}-\rho_{Y_{t}^{\prime}}\right) \ln \rho_{Y_{t}}\right\} .
$$

The entropy flux is seen to depend solely on the degrees of freedom of the ancilla. Although Eq. (26) is general and holds for arbitrary states of the ancillae, it reduces to $\beta \dot{Q}$, as in the Clausius expression, if $\rho_{Y}$ is thermal.

Another very important property of the entropy flux is additivity. What we call an "ancilla" may itself be a composed system consisting of multiple elementary units. In fact, as we illustrate in Sec. IV, this can give rise to interesting situations. Suppose that $Y_{t}=\left(Y_{t 1}, Y_{t 2}, \ldots, Y_{t N}\right)$ and that the units are prepared in a globally product state $\rho_{Y_{t}}=\bigotimes_{j=1}^{N} \rho_{Y_{t j}}$. After colliding with the system, the state $\rho_{Y_{t}^{\prime}}$ might no longer be uncorrelated, in general. Despite this, owing to the structure of Eq. (26), we would have

$$
\Delta \Phi_{t}^{u}=\sum_{j=1}^{N} \Delta \Phi_{t j}^{u}=\sum_{j=1}^{N} \operatorname{tr}\left\{\left(\rho_{Y_{t j}^{\prime}}-\rho_{Y_{t j}}\right) \ln \rho_{Y_{t j}}\right\},
$$

where $\rho_{Y_{t j}^{\prime}}$ is the postcollision reduced state of the $j$ th unit of the ancilla. This property is quite important, as it allows one to compute the flux associated with each dissipation channel acting on the system.

\section{Conditional second law}

Equations (22), (23), and (26) specify the thermodynamics of the unconditional trajectories $\rho_{X_{t}}$, when no information about the ancillae is recorded. We now ask the same question for the conditional trajectories $\rho_{X_{t} \mid \zeta_{t}}$. In this case, the relevant entropy is the quantum-classical conditional entropy $S\left(X_{t} \mid \zeta_{t}\right)$ in Eq. (10). Thus, we search for a splitting analogous to Eq. (22), but of the form

$$
\Delta \Sigma_{t}^{c}=S\left(X_{t} \mid \zeta_{t}\right)-S\left(X_{t-1} \mid \zeta_{t-1}\right)+\Delta \Phi_{t}^{c}
$$

where $\Delta \Sigma_{t}^{c}$ and $\Delta \Phi_{t}^{c}$ are the conditional counterparts of the unconditional quantities used in Sec. III C. The identification of suitable forms for such quantities is the scope of this section.

We adopt an approach similar to that used in Refs. [20, 56], which consists in defining the conditional flux rate as the natural extension of Eq. (26) to the conditional case. That is, as $\Delta \Phi_{t}^{c}$ refers to a specific collision, it should depend only on quantities pertaining to the specific ancilla $Y_{t}$, thus being of the form

$$
\Delta \Phi_{t}^{c}=S\left(Y_{t}^{\prime} \mid z_{t}\right)-S\left(Y_{t}\right)+\sum_{z_{t}} P\left(z_{t}\right) D\left(\rho_{Y_{t}^{\prime} \mid z_{t}}|| \rho_{Y_{t}}\right),
$$

where $\rho_{Y_{t}^{\prime}} \mid z_{t}=\left(M_{z_{t}} \rho_{Y_{t}^{\prime}} M_{z_{t}}^{\dagger}\right) / P\left(z_{t}\right)$ is the final state of the ancilla given outcome $z_{t}$ and $P\left(z_{t}\right)=\operatorname{tr}\left(M_{z_{t}} \rho_{Y_{t}^{\prime}} M_{z_{t}}^{\dagger}\right)$ [cf. Eq. (3)]. Moreover, $S\left(Y_{t}^{\prime} \mid z_{t}\right)$ is defined similarly to Eq. (10). Note how the causal structure of the model implies that the flux should be conditioned only to outcome $z_{t}$, instead of the entire measurement record $\zeta_{t}$.

By defining the reconstructed state of the ancilla after the measurement $\tilde{\rho}_{Y_{t}^{\prime}}=\sum_{z_{t}} P\left(z_{t}\right) \rho_{Y_{t}^{\prime} \mid z_{t}}=\sum_{z_{t}} M_{z_{t}} \rho_{Y_{t}^{\prime}} M_{z_{t}}^{\dagger}$, Eq. (29) can be recast into the form

$$
\Delta \Phi_{t}^{c}=\operatorname{tr}\left\{\left(\rho_{Y_{t}}-\tilde{\rho}_{Y_{t}^{\prime}}\right) \ln \rho_{Y_{t}}\right\},
$$

which showcases the potential difference between conditional and unconditional fluxes. Depending on the measurement strategy $\left\{M_{z}\right\}$ being adopted, it is reasonable to expect that $\rho_{Y_{t}^{\prime}} \neq \tilde{\rho}_{Y_{t}^{\prime}}$, thus resulting in $\Delta \Phi_{t}^{u} \neq \Delta \Phi_{t}^{c}$. This reflects the potentially invasive nature of the measurements on the ancilla. However, it should be noted that this is an extrinsic effect, related to the specific choice of measurement by the observer, and fully unrelated to the thermodynamics of the system-ancilla interactions.

We henceforth assume that the measurement strategy is such that

$$
\operatorname{tr}\left\{\tilde{\rho}_{Y_{t}^{\prime}} \ln \rho_{Y_{t}}\right\}=\operatorname{tr}\left\{\rho_{Y_{t}^{\prime}} \ln \rho_{Y_{t}}\right\} .
$$

That is, it does not change the population of $Y_{t}^{\prime}$ in the eigenbasis of the original state $\rho_{Y_{t}}$. This can be accomplished, 
for instance, by measuring in the same basis into which the state of the ancillae is prepared. We can then reach the important conclusion that

$$
\Delta \Phi_{t}^{c}=\Delta \Phi_{t}^{u}
$$

This result is intuitive: conditioning on the outcome is a subjective matter related to whether or not we read out the outcomes of the experiment. It should therefore have no effect on how much entropy flows to the ancillae. Similar ideas were also used in many contexts [10,12,18,56,57]. However, these studies were concerned with the heat flux, which coincides with the entropy flux for thermal baths. Here we show that this is a general property, valid for any bath, provided we restrict to the special class of measurements characterized by Eq. (31).

Under these conditions, comparing Eqs. (28) and (22), and recalling the information rate in Eq. (15), we find that

$$
\Delta \Sigma_{t}^{c}=\Delta \Sigma_{t}^{u}-\Delta I_{t}
$$

This is a key result of our framework: It shows how the act of conditioning the dynamics on the measurement outcome changes the entropy production by a quantity associated with the change in the Holevo information. Hence, it serves as a bridge between the information rates and thermodynamics. In particular, in an ISS, $\Delta I_{\text {ISS }}=0$ and so $\Delta \Sigma_{\text {ISS }}^{c}=$ $\Delta \Sigma_{\text {ISS }}^{u}$, although $\rho_{X_{t}}$ and $\rho_{X_{t} \mid \zeta_{t}}$ are in general different.

\section{E. Properties of the conditional entropy production}

We now move on to discuss the main properties of the conditional entropy production. The quantities $\Delta \Sigma_{t}^{u}$ and $\Delta \Sigma_{t}^{c}$ refer to the incremental entropy production in a single collision. Conversely, it is also of interest to analyze the integrated entropy production

$$
\Sigma_{t}^{\alpha}=\sum_{\tau=1}^{t} \Delta \Sigma_{\tau}^{\alpha}, \quad \alpha=u, c .
$$

Since $\Delta I_{t}$ in Eq. (15) is an exact differential, when we sum Eq. (33) up to time $t$, the terms in $\Delta I_{\tau}$ successively cancel, leaving only

$$
\Sigma_{t}^{c}=\Sigma_{t}^{u}-I\left(X_{t}: \zeta_{t}\right)
$$

The integrated entropy production up to time $t$ therefore depends only on the net information $I\left(X_{t}: \zeta_{t}\right)$. Since $I\left(X_{t}: \zeta_{t}\right) \geq 0$, it then follows that

$$
\Sigma_{t}^{u} \geq \Sigma_{t}^{c}
$$

Therefore,conditioning reduces the entropy produced in the dynamics. This happens because we only carry out measurements in the environment, so that there is never a direct backaction in the system. An upper bound to the difference of the two entropy productions can also be obtained by using the fact that $L_{t} \geq 0$, which then leads to

$$
\Sigma_{t}^{u}-\Sigma_{t}^{c} \leq \sum_{\tau=1}^{t} G_{\tau} .
$$

The reduction in entropy production is thus at most the total information gain.

Returning now to the entropy production rate in each collision, in Appendix B we provide a proof of the relation

$$
\Delta \Sigma_{t}^{c} \geq D\left(Y_{t}^{\prime} \| Y_{t}\right)+I\left(Y_{t}^{\prime}: \zeta_{t}\right) \geq 0
$$

where $D\left(Y_{t}^{\prime} \| Y_{t}\right)$ is the backaction caused in the ancillary state due to its collision with the system, while $I\left(Y_{t}^{\prime}: \zeta_{t-1}\right)$ quantifies the amount of information gained about the ancilla through the measurement strategy. This is one of the overarching conclusions of our work, bearing remarkable consequences. On the one hand, it proves that the second law continues to be satisfied in the conditional case. On the other hand, it provides a nontrivial lower bound to the conditional entropy production rate in terms of the changes that take place in the ancillae only. It should also be noted that the first inequality in Eq. (38) is saturated by processes where the measurement extracts all the information available.

\section{SIMPLE QUBIT MODELS}

We now apply the ideas of the previous sections to simple models of $\mathrm{CM}^{2} \mathrm{~s}$, aimed at illustrating their overarching features while keeping the level of technical details to a minimum, so as to emphasize the physical implications of the framework illustrated so far.

We focus on the case in which both the system and the elementary units of the ancilla are qubits. Despite their simplicity, such situations have far-reaching applications. For instance, in Ref. [45] it was shown how quantum optical stochastic master equations naturally emerge from modeling optical baths in terms of effective qubits in a collisional model. Moreover, suitably chosen measurement strategies $\left\{M_{z}\right\}$ implemented on qubits also allow one to simulate widely used measurement schemes, such as photodetection and homodyne and heterodyne measurements. Finally, by tuning the initial state of the qubits, one can also simulate out-of-equilibrium environments, such as squeezed baths. In Ref. [58], we complement the study reported here by addressing explicitly the case of continuous-variable systems.

Recall that a $\mathrm{CM}^{2}$ is completely specified by setting $\left\{\rho_{Y}, U, M_{z}\right\}$. The unconditional dynamics is governed by the map $\mathcal{E}$ defined in Eq. (2), which can be simulated directly with very low computational cost. The conditional dynamics, on the other hand, is governed by the map $\mathcal{E}_{z}$ in Eq. (8), which we simulate using stochastic trajectories. 


\section{A. Single-qubit ancilla}

We begin by studying the case where the system interacts with single-qubit ancillae prepared in the thermal state $\rho_{Y}=f|0\rangle\left\langle\left. 0\right|_{Y}+(1-f) \mid 1\right\rangle\left\langle\left. 1\right|_{Y}\right.$, where $f \in$ $[0,1]$ and $|0\rangle,|1\rangle$ is the computational basis - i.e., the eigenstates of the Pauli-z operator $\sigma_{Y}^{z}=|1\rangle\left\langle\left. 1\right|_{Y}-\mid 0\right\rangle\left\langle\left. 0\right|_{Y}\right.$. The collisions are modeled by a partial SWAP gate $U=e^{-i g\left(\sigma_{X}^{+} \sigma_{Y}^{-}+\sigma_{X}^{-} \sigma_{Y}^{+}\right)}$, where $\sigma_{\alpha}^{+}=\left(\sigma_{\alpha}^{-}\right)^{\dagger}=|1\rangle\left\langle\left. 0\right|_{\alpha}\right.$ is the Pauli raising operator $(\alpha=X, Y)$. Finally, we assume that the ancillae are measured in the computational basis, so that $M_{0}=|0\rangle\left\langle\left. 0\right|_{Y}\right.$ and $\left.M_{1}=\mid 1\right\rangle\left\langle\left. 1\right|_{Y}\right.$. For concreteness, we take the initial state of the system to be $\rho_{X_{0}}=\left|x_{+}\right\rangle\left\langle\left. x_{+}\right|_{X}\right.$, where $\sigma_{X}^{x}\left|x_{+}\right\rangle_{X}=\left|x_{+}\right\rangle_{X}$.

The evolution of the relevant information and thermodynamic quantities of the problem, for a specific choice of $f$ and $g$, is presented in Fig. 2. Panel (b) shows how conditioning always reduces our ignorance about the system, by demonstrating that $S\left(X_{t} \mid \zeta_{t}\right) \leq S\left(X_{t}\right)$ at all times. A property of this model is that the unconditional steady state $\rho_{X_{\infty}}$ always coincides with the initial state of the ancilla, $\rho_{X_{\infty}}=\rho_{Y}$, a property known as homogenization [32,33]. This causes $U\left(\rho_{X_{\infty}} \otimes \rho_{Y}\right) U^{\dagger}=\rho_{X_{\infty}} \otimes \rho_{Y}$, so no information can be acquired anymore. The final state is thus an equilibrium state, not an ISS. The information rate, gain, and loss are shown in Fig. 2(c). Initially, the gain is very large, as the state of the system is significantly different from the thermal steady state and each measurement results in a significant acquisition of information. In turn, this results in $\Delta I_{t}>0$. As the system evolves towards
$\rho_{X_{\infty}}$, the detrimental effect of homogenization starts prevailing over the information gain, causing an inversion in the sign of $\Delta I_{t}$. The long-time limit is associated with $\left(\Delta I_{\infty}, G_{\infty}, L_{\infty}\right) \rightarrow 0$ and no ISS emerges.

A comparison between the conditional and unconditional entropy productions is shown in Fig. 2(d), which also reports on the entropy flux. The rates $\Delta \Sigma_{t}^{c}$ and $\Delta \Sigma_{t}^{u}$ are both non-negative, but are not necessarily ordered. This happens because, in individual collisions, conditioning may not reduce the entropy produced. An ordering is instead enforced when looking at integrated quantities: conditioning always reduces the entropy production [cf. Eq. (35)], as shown in Fig. 2(e).

For completeness, we also show in Figs. 2(f)-2(h) the behaviors of $\Delta I_{t}, G_{t}$, and $L_{t}$ along six randomly sampled trajectories $\zeta_{t}$. Typical stochastic fluctuations are observed, showing that in a single stochastic run, the net gain and loss can differ substantially [the curves in Figs. 2(b)-2(e) are produced by averaging over 2000 such trajectories].

\section{B. Two-qubit ancilla}

We now move on to consider a case allowing the emergence of ISSs, opening up many interesting possibilities. The ancillae do not have to be just a single qubit, but can have arbitrary internal structure. Moreover, within a single collision, the system does not have to interact with all elementary units simultaneously, but may do so sequentially. We illustrate this by considering the case where each (a)

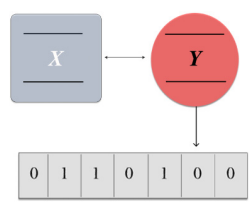

(e)

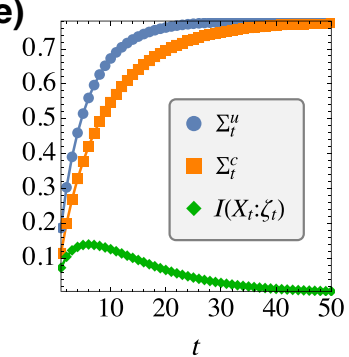

(b)

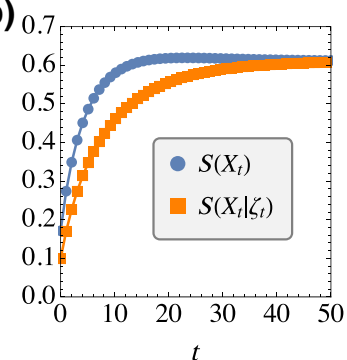

(f)

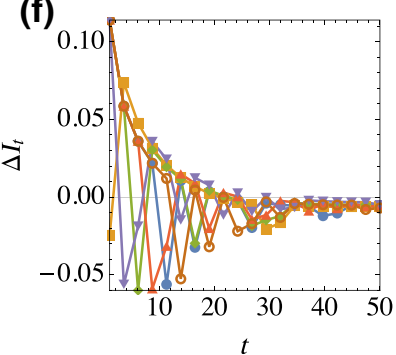

(c)

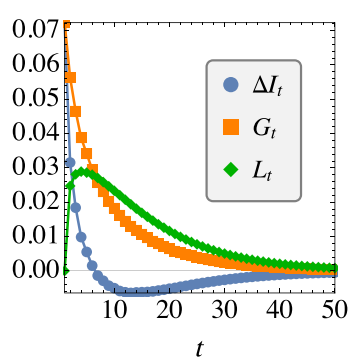

(g)

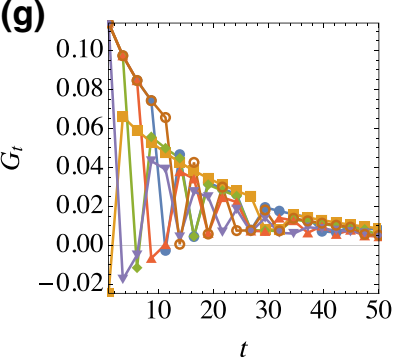

(d)

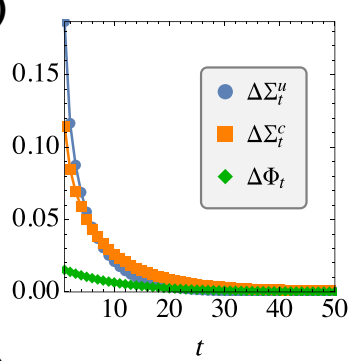

(h)

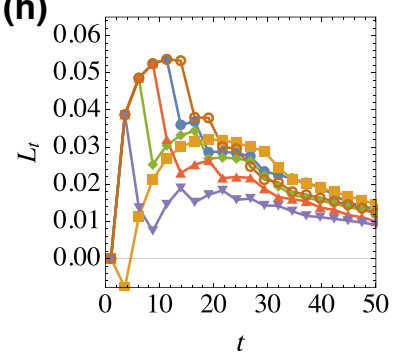

FIG. 2. (a) Dynamics of a $\mathrm{CM}^{2}$ under a quantum homogenization process where both the system and ancilla are qubits. (b) Unconditional and conditional entropies. (c) Information rate $\Delta I_{t}$ [Eq. (15)], and its splitting into a gain and loss term [Eq. (16)]. (d) Unconditional and conditional entropy production rates, $\Delta \Sigma_{t}^{u}$ and $\Delta \Sigma_{t}^{c}$, as well as the entropy flux $\Delta \Phi_{t}$. (e) Integrated unconditional and conditional entropy productions, and net Holevo information $I\left(X_{t}: \zeta_{t}\right)$ [cf. Eq. (35)]. (f),(g),(h) Sample stochastic trajectories of $\Delta I_{t}, G_{t}$, and $L_{t}$. We have taken $f=g=0.3$ (the results do not depend qualitatively on such choices), while details on how we chose $\rho_{Y}, \rho_{X_{0}}, U$, and $\left\{M_{z}\right\}$ are explained in the main text. 
(a)

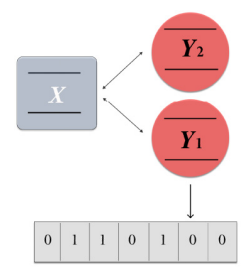

(e)

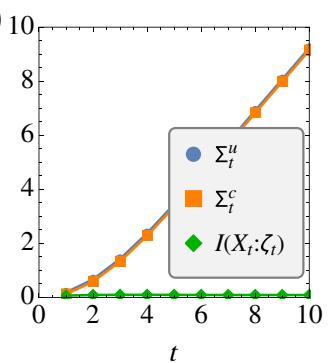

(b)

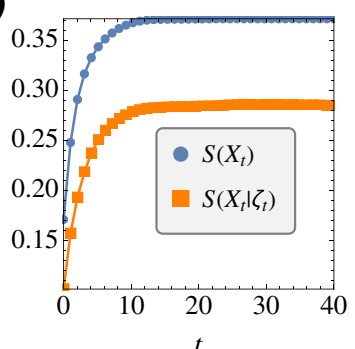

(f)

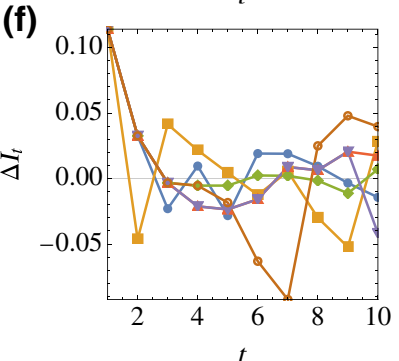

(c)

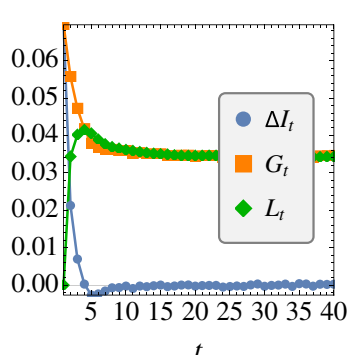

(g)

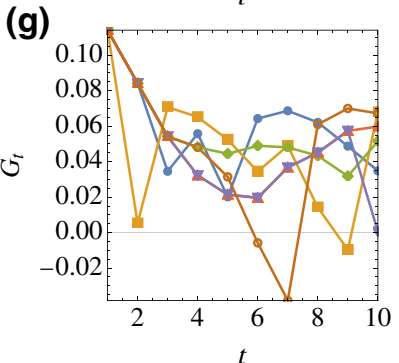

(d)

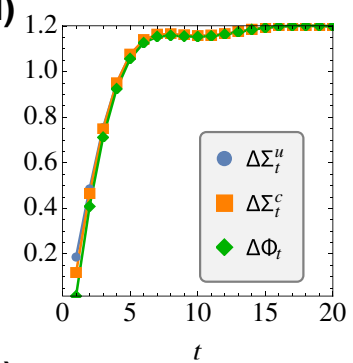

(h)

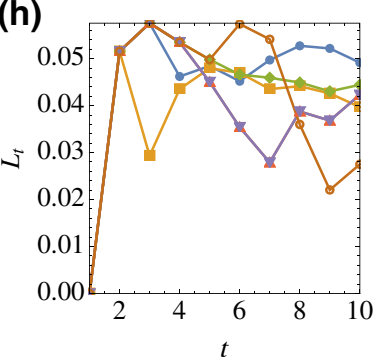

FIG. 3. Same as Fig. (2), but for two-qubit ancillae, prepared in $\rho_{Y_{1}}=f|0\rangle\langle 0|+(1-f)| 1\rangle\langle 1|$ and $\rho_{Y_{2}}=\left|x_{+}\right\rangle\left\langle x_{+}\right|$. The qubits interact sequentially with the system via partial SWAP gates and only ancilla $Y_{1}$ is measured. In contrast to Fig. (2), this model has a nontrivial ISS ( $G=L \neq 0$ ). We have taken, for concreteness, $f=g_{1}=0.3$ and $g_{2}=0.5$.

ancilla is actually two qubits, $Y_{t}=\left(Y_{t 1}, Y_{t 2}\right)$, which interact sequentially with the system (cf. Fig. 3). The unitary $U_{t}$ between $X$ and $Y_{t}$ will then have the form

$$
U_{t}=U_{X Y_{t 2}} U_{X Y_{t 1}}
$$

where $U_{X Y_{t j}}$ has support only over the Hilbert space of $X$ and the unit $Y_{t j}$. As discussed in Refs. [28,41], if the ancillae are prepared in different states, the system will not be able to equilibrate with either, but will instead keep on bouncing back and forth indefinitely. Hence, it will reach a NESS. Moreover, if at least one of the ancillae are measured, the conditional state may embody an ISS.

To illustrate this, we assume the first unit to be prepared in a thermal state such as that considered in Sec. IV A, while the second unit is in $\left|x_{+}\right\rangle$. The unitaries in Eq. (39) are chosen, as before, to be partial SWAP gates with strengths $g_{1}$ and $g_{2}$. Finally, we choose to measure only the first unit that, by being prepared in a thermal state, acts as a classical probe. On the other hand, by being endowed with quantum coherence, the second unit represents a "resourceful state."

In Fig. 3 we report the results of an analysis similar to that performed for the previous example, for direct comparison. The results are strikingly different as, in particular, the system now allows for an ISS. This is visible in Fig. 3(c) from the fact that $G=L \neq 0$ when $t \rightarrow \infty$ with the thermodynamic quantities in Fig. 3(d) also converging to nonzero long-time values. A marked difference with the case of no ISS is also seen in the behavior of the integrated entropy production in Fig. 3(e): as the rates now remain nonzero, the integrated quantities diverge in the long-time limit.

We can also perform another experiment that beautifully illustrates the essence of an ISS. While the initial state used in Fig. 3 was arbitrarily chosen, we could take it to be the steady state of the unconditional dynamics. The idea is that we first allow the system to unconditionally (a)

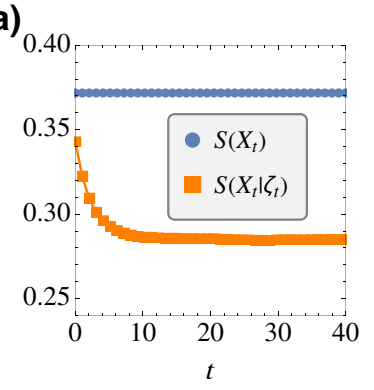

(b)

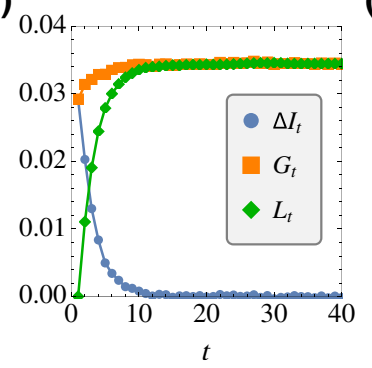

(c)

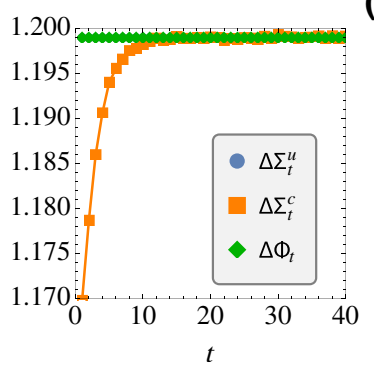

(d)

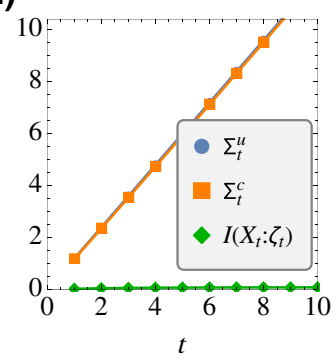

FIG. 4. Similar to the two-qubit scenario of Figs. 3(b)-3(e), but with the initial state $\rho_{X_{0}}$ chosen as the fixed point $\rho_{X} *$ of the unconditional dynamics. 
(a)

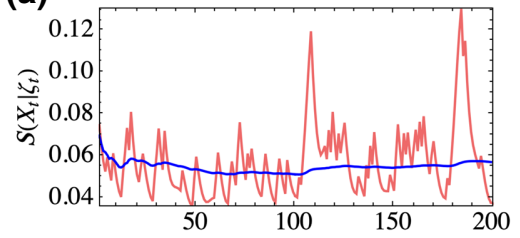

(c)

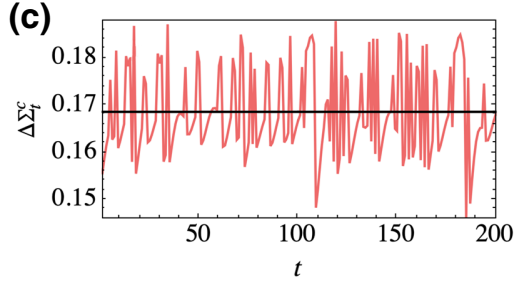

(b)

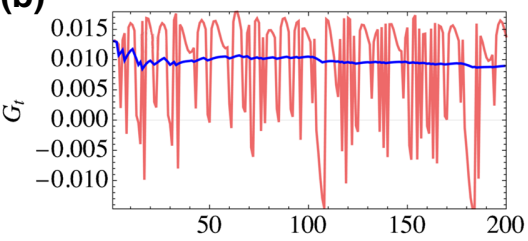

(d)

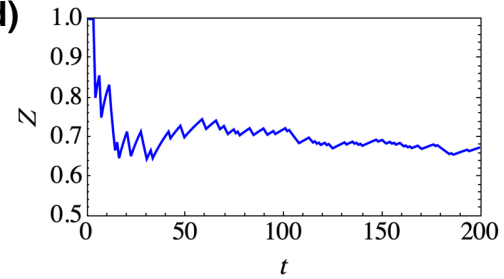

(e)

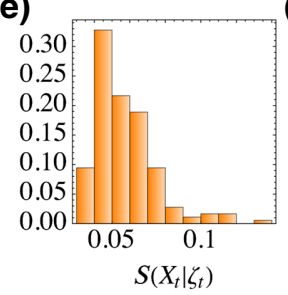

(g)

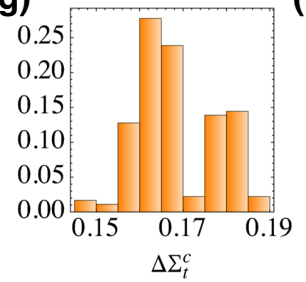

(f)

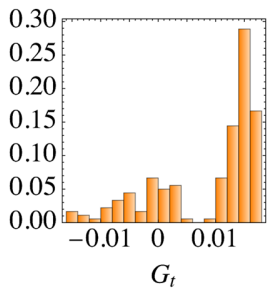

(h)

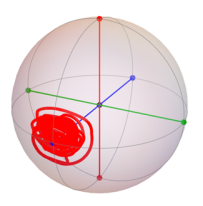

FIG. 5. Thermodynamics and information in the single-shot scenario. The configuration is the same as Fig. 4, but everything now refers to a single stochastic realization of the experiment. The red curves depict (a) $S\left(X_{t} \mid \zeta_{t}\right)$, (b) $G_{t}$, and (c) $\Delta \Sigma_{t}^{c}$ for that single realization. The blue curves, on the other hand, represent the accumulated average; that is, the average of the given quantity up to that time. Image (d), in particular, shows the accumulated average for the outcomes $Z_{t}=\left(\sum_{j=1}^{t} z_{t}\right) / t$, where the outcomes $z_{t}$ are either 0 or 1 (not shown for visibility). The black line in image (c) is the unconditional entropy production rate $\Delta \Sigma_{t}^{u}$, which serves as a baseline for $\Delta \Sigma_{t}^{c}$. Images (e)-(g) are the histograms obtained from the data in (a)-(d), discarding the first 20 points (to eliminate transients). (h) Stochastic trajectory in Bloch's sphere.

relax by letting it undergo a large number of collisions, and only then we start measuring. Because of the effect of the measurements, the conditional state $\rho_{X_{t} \mid \zeta t}$ will start to differ from the unconditional steady state (while the unconditional dynamics remains fixed).

The results are shown in Fig. 4. Panel (a), in particular, neatly illustrates how the unconditional entropy does not change in time, while the measurements performed in the conditional strategy reduce the entropy of the state of the system, which is effectively driven to a state with a larger purity. This is the essence of an ISS.

In the last two examples, the ancillae are measured in the same basis in which they are initially prepared. An ISS is expected to exist even if this assumption is lifted. However, in this case Eq. (32) will no longer hold, in general, and the additional entropy flux generated by the measurement backaction must be explicitly taken into account in the thermodynamics of the system.

\section{Time series in the single-shot scenario}

The quantities in Figs. 2-4 are obtained by repeating the experiment multiple times, always starting from the same state and evolving in the exact same way. We now contrast this with the single-shot scenario. That is, when we have access only to a single stochastic realization of the experiment. We focus on the two-qubit model where the system starts in the steady state of the unconditional dynamics, as in Fig. 4. The dynamics of $S\left(X_{t} \mid \zeta_{t}\right), G_{t}$, and $\Delta \Sigma_{t}^{c}$ along a single trajectory are shown in Fig. 5. As one might expect, these quantities fluctuate significantly.
Figure 5 also shows the behavior of accumulated averages, up to a certain time, showing that both the entropy and gain rate tend to converge precisely to the ISS value in Fig. 4. In a classical context, processes satisfying this property are called stationary ergodic [59]. In Fig. 5(d) we plot the integrated average of the actual outcomes, $Z_{t}=\left(\sum_{j=1}^{t} z_{t}\right) / t$, the actual outcomes being binary. Such integrated average outcome shows that in the ISS $70 \%$ of the clicks are associated with $M_{1}$ and the remaining 30\% with $M_{0}$.

Finally, the single-shot data in Figs. 5(a)-5(d) can also be used to construct a histogram of the most relevant quantities, as illustrated in panels (e)-(h). These histograms shed light on the magnitude of the fluctuations of the relevant quantities. For instance, $\Delta \Sigma_{t}^{c}$ fluctuates very little, while the information gain $G_{t}$ fluctuates dramatically.

\section{CONCLUSIONS}

We have investigated the interplay between information and thermodynamics in continuously measured system by way of a collisional model construct. The latter offers a flexible framework to tackle information-thermodynamic issues and allowed us to generalize recent results [20,27] beyond Gaussian systems and dynamics. Far from trivial, this generalization opens the possibility of applying our results to generic quantum systems and dynamics. More importantly, the results that we have obtained allow for a formal characterization of information-theoretic quantities. In particular, we were able to formulate the entropy production and flux rate - two pivotal quantities in (quantum) thermodynamics - from a purely informational point of 
view and account for repeated indirect measurements of the system of interest. These results offer a clear way to point out and characterize the effect of quantum measurements on the thermodynamics of open quantum system.

We model the indirect measurement of the system via a collisional model where (a part of) the environment with which the system interacts is monitored. This allows us to compare the entropy production with the case in which the environment is not measured and the evolution of the system is thus unconditioned. In turn, this comparison leads directly to a tightened second law for monitored systems with a very clear separation between entropic contributions coming from the dissipative interaction with the environment and those coming from the information gained during the monitoring. This allows us to introduce the concept of information gain and loss rates, and informational steady states. The latter are particularly interesting since they represent cases where a delicate balance is established between the information that gets lost into the environment and that which is extracted by measuring.

The interplay between information and the second law has been the subject of several works over the last decade. Stroboscopic dynamics, such as that considered in Sec. II, has been studied in the classical context of hidden Markov models $[9,48,49]$. A classical framework, where quantum measurements are mimicked by generic interventions, was put forth in Ref. [18], and resembles the classical version of our $\mathrm{CM}^{2} \mathrm{~s}$, developed in Appendix $\mathrm{A}$. In the quantum context, the conditional dynamics analyzed here are a particular case of process tensors [60-62] whose thermodynamics has recently been considered in Refs. [19,63]. Unlike our framework, however, these studies assume that the system is always connected to a standard thermal bath, while the ancillae play only the role of memory agents. For this reason, their definition of entropy production is based on a Clausius-like inequality and is therefore different from ours. Furthermore, we have opted to focus on informational aspects of thermodynamics, neglecting entirely the energetics of the problem. Detailed accounts of the latter can be found in Refs. [19,57,63,64].

Funo et al. [12] put forth a framework (recently assessed experimentally in Ref. [65]) where the ancillae play the role of active memories. This means that their effect is always deleterious to the system. As a consequence, instead of using the Holevo quantity (11) to quantify information, they used the Groenewold-Ozawa quantumclassical information $[66,67] I_{\mathrm{GO}}=S(X)-S\left(X^{\prime} \mid z\right)$. The two quantities are related by $I\left(X^{\prime}: z\right)=I_{\mathrm{GO}}-\Delta S_{X}$, where $\Delta S_{X}=S\left(X^{\prime}\right)-S(X)$. Depending on the type of collision, $\Delta S_{X}$ may have any sign, so $I_{\mathrm{GO}}$ is not necessarily non-negative.

The formalism developed in this work is widely applicable, as exemplified by the case studies we have considered (see also Ref. [58]). This makes it a valuable tool in the thermodynamic assessment of a broad variety of quantum-coherent experiments. The scenario we considered also fits perfectly with the characterization of emergent quantum applications, such as quantum computing devices [68-70]. Being able to characterize irreversibility in these devices should thus offer a significant advantage in the design and engineering of future devices.

\section{ACKNOWLEDGMENTS}

We acknowledge support from the Deutsche Forschungsgemeinschaft (DFG, German Research Foundation) project number BR 5221/4-1, the MSCA project pERFEcTO (Grant No. 795782), the H2020-FETOPEN-2018-2020 TEQ (Grant No. 766900), the DfE-SFI Investigator Programme (Grant No. 15/IA/2864), COST Action CA15220, the Royal Society Wolfson Research Fellowship (RSWF $\backslash$ R3 $\backslash 183013$ ), the Leverhulme Trust Research Project Grant (Grant No. RGP-2018-266), and the UK EPSRC (Grant No. EP/T028106/1).

\section{APPENDIX A: CLASSICAL (INCOHERENT) CM $^{2}$}

It is interesting to enquire what the classical analogs of the quantum model put forth in Sec. II are. Or, put differently, what are the conditions for the model to be called classical, or incoherent?

Let us focus on a single collision event. We assume that, at a certain instant of time, the system is at $\rho_{X}=$ $\sum_{x} p(x)|x\rangle\langle x|$ for some basis $|x\rangle$, while the ancilla is prepared in $\rho_{Y}=\sum_{y} p(y)|y\rangle\langle y|$ for some basis $|y\rangle$. The unconditional state of the system after one collision will then be

$$
\rho_{X}^{\prime}=\mathcal{E}\left(\rho_{X}\right)=\sum_{x, y, y^{\prime}} p(x) p(y)\left\langle y^{\prime}|U| x y\right\rangle\left\langle x y\left|U^{\dagger}\right| y^{\prime}\right\rangle,
$$

where $\left\langle y^{\prime}|U| x y\right\rangle$ is still a ket in the Hilbert space of the system. This ket is not normalized, however, so we define

$$
\left|\Psi_{x y y^{\prime}}\right\rangle:=\frac{\left\langle y^{\prime}|U| x y\right\rangle}{\sqrt{P\left(y^{\prime} \mid x y\right)}}, \quad P\left(y^{\prime} \mid x y\right)=\left\|\left\langle y^{\prime}|U| x y\right\rangle\right\|^{2} .
$$

The state of the system may then be written as

$$
\rho_{X}^{\prime}=\sum_{x y y^{\prime}} p(x) p(y) P\left(y^{\prime} \mid x y\right)\left|\Psi_{x y y^{\prime}}\right\rangle\left\langle\Psi_{x y y^{\prime}}\right| .
$$

When written in this way, it gives the impression that $\rho_{X}^{\prime}$ is already in diagonal form. But this is not the case, since, in general, the states $\left|\Psi_{x y y^{\prime}}\right\rangle$ are not orthogonal and do not form a basis. Moreover, there are usually many more states than that required to span the Hilbert space of $X$ (there can be up to $d_{X} d_{Y}^{2}$ of them, where $d_{X}, d_{Y}$ are the dimensions of the system and ancilla). As a matter of fact, in general, the 
eigenvectors of $\rho_{X}^{\prime}$ will have no simple relation with the states $\left|\Psi_{x y y^{\prime}}\right\rangle$.

Conversely, we say a model is unconditionally incoherent if, for any $x y y^{\prime}$, the states $\left|\Psi_{x y y^{\prime}}\right\rangle$ are always elements of the basis $|x\rangle$. In this case $\rho_{X}^{\prime}$ will be automatically diagonal,

$$
\rho_{X}^{\prime}=\sum_{x^{\prime}} p\left(x^{\prime}\right)\left|x^{\prime}\right\rangle\left\langle x^{\prime}\right|
$$

where the populations $p\left(x^{\prime}\right)$ can be found from

$$
\begin{aligned}
p\left(x^{\prime}\right) & =\left\langle x^{\prime}\left|\rho_{X}^{\prime}\right| x^{\prime}\right\rangle \\
& =\sum_{x, y, y^{\prime}} p(x) p(y) P\left(y^{\prime} \mid x y\right)\left\langle x^{\prime} \mid \Psi_{x y y^{\prime}}\right\rangle\left\langle\Psi_{x y y^{\prime}} \mid x^{\prime}\right\rangle .
\end{aligned}
$$

Using Eq. (A1), we can also write this as

$$
p\left(x^{\prime}\right)=\sum_{x, y, y^{\prime}} Q\left(x^{\prime} y^{\prime} \mid x y\right) p(x) p(y),
$$

where

$$
Q\left(x^{\prime} y^{\prime} \mid x y\right)=\left|\left\langle x^{\prime} y^{\prime}|U| x y\right\rangle\right|^{2}
$$

is the transition probability of observing a transition $(x, y) \rightarrow\left(x^{\prime}, y^{\prime}\right)$. A matrix of this form is said to be unistochastic, which is a particular case of doubly stochastic matrices.

An example of an unconditionally incoherent model is when both the system and ancillae are qubits, interacting with the partial SWAP gate

$$
\begin{aligned}
U= & (|00\rangle\langle 00|+| 11\rangle\langle 11|)+\lambda(|01\rangle\langle 01|+| 10\rangle\langle 10|) \\
& -i \sqrt{1-\lambda^{2}}(|01\rangle\langle 10|+| 10\rangle\langle 01|) .
\end{aligned}
$$

In this case

$$
Q=\left(\begin{array}{cccc}
1 & 0 & 0 & 0 \\
0 & \lambda^{2} & 1-\lambda^{2} & 0 \\
0 & 1-\lambda^{2} & \lambda^{2} & 0 \\
0 & 0 & 0 & 1
\end{array}\right)
$$

with $\lambda \in[0,1]$.

In unconditionally incoherent models, if the system is originally diagonal in the basis $|x\rangle$, it will remain so throughout the evolution, with the populations evolving according to the classical Markov chain

$$
\begin{aligned}
& p\left(x_{t+1}\right)=\sum_{x_{t}} \mathcal{Q}\left(x_{t+1} \mid x_{t}\right) p\left(x_{t}\right) \\
& \mathcal{Q}\left(x^{\prime} \mid x\right)=\sum_{y, y^{\prime}} Q\left(x^{\prime} y^{\prime} \mid x y\right) p(y)
\end{aligned}
$$

Next we can do the same for the conditional map $\mathcal{E}_{z}$ in Eq. (8). As we will see, however, unconditional incoherence does not imply conditional incoherence. Following the same steps as before, we can write

$$
\mathcal{E}_{z}\left(\rho_{X}\right)=\sum_{x y y^{\prime}} p(x) p(y)\left\langle y^{\prime}\left|M_{z} U\right| x y\right\rangle\left\langle x y\left|U^{\dagger} M_{z}^{\dagger}\right| y^{\prime}\right\rangle .
$$

We now introduce two completeness relations in the $y$ basis:

$$
\begin{aligned}
\mathcal{E}_{z}\left(\rho_{X}\right)= & \sum_{x y y^{\prime} y^{\prime \prime} y^{\prime \prime \prime}} p(x) p(y)\left\langle y^{\prime}\left|M_{z}\right| y^{\prime \prime}\right\rangle\left\langle y^{\prime \prime}|U| x y\right\rangle \\
& \times\left\langle x y\left|U^{\dagger}\right| y^{\prime \prime \prime}\right\rangle\left\langle y^{\prime \prime \prime}\left|M_{z}^{\dagger}\right| y^{\prime}\right\rangle .
\end{aligned}
$$

If the model is unconditionally incoherent, the states $\left\langle y^{\prime \prime}|U| x y\right\rangle$ will be elements of the basis $|x\rangle$. But the resulting state will in general not be diagonal due to the terms $\left\langle y^{\prime}\left|M_{z}\right| y^{\prime \prime}\right\rangle$ and $\left\langle y^{\prime \prime \prime}\left|M_{z}\right| y^{\prime}\right\rangle$. In other words, coherence may very well be produced by the measurement itself. And while this cannot affect the unconditional dynamics of the system (due to no signaling), it may very well affect the conditional one.

We therefore define a model to be conditionally incoherent if it is unconditionally incoherent and if

$$
\left\langle y^{\prime}\left|M_{z}\right| y^{\prime \prime}\right\rangle \propto \Delta_{y^{\prime}, y^{\prime \prime}}
$$

The simplest possibility would, of course, be to take $M_{z}$ as projective measurements in the basis $|y\rangle$. But there may also be other interesting possibilities. For instance, we can take $M_{z}$ to be an imprecise projective measurement, which only runs over certain elements of the basis $|y\rangle$. Or we could make $M_{z}$ be a noisy measurement that blurs the outcomes of each $|y\rangle$. It is worth noting, in passing, that conditional incoherence also immediately implies the validity of Eq. (32) on the entropy fluxes for conditionally incoherent models.

In any case, when the model is conditionally incoherent, the map (8) can be written as

$$
\mathcal{E}_{z}\left(\rho_{X}\right)=\sum_{x, y, y^{\prime}} p(x) p(y) M\left(z \mid y^{\prime}\right) P\left(y^{\prime} \mid x y\right)\left|\Psi_{x y y^{\prime}}\right\rangle\left\langle\Psi_{x y y^{\prime}}\right|
$$

where

$$
M\left(z \mid y^{\prime}\right)=\left|\left\langle y^{\prime}\left|M_{z}\right| y^{\prime}\right\rangle\right|^{2}=\operatorname{tr}\left[M_{z}^{\dagger} M_{z}\left|y^{\prime}\right\rangle\left\langle y^{\prime}\right|\right]
$$

is the conditional probability of observing outcome $z$, given that the ancilla is in $\left|y^{\prime}\right\rangle$. This therefore represents the "postprocessing" of the ancillary state. State (A8) can 
also be written as

$$
\mathcal{E}_{z}\left(\rho_{X}\right)=\sum_{x^{\prime}} p\left(x^{\prime}, z\right)\left|x^{\prime}\right\rangle\left\langle x^{\prime}\right|,
$$

where

$$
p\left(x^{\prime}, z\right)=\sum_{x, y, y^{\prime}} p(x) p(y) M\left(z \mid y^{\prime}\right) Q\left(x^{\prime} y^{\prime} \mid x y\right) .
$$

This is consistent with Eq. (9): since the result of the map is a distribution in both $x^{\prime}$ and $z$, if we trace over $X$, we are left only with $p(z)$.

At this point it is convenient to define the transition matrix

$$
W\left(x^{\prime} z \mid x\right)=\sum_{y, y^{\prime}} M\left(z \mid y^{\prime}\right) Q\left(x^{\prime} y^{\prime} \mid x y\right) p(y) .
$$

In a classical context, this is the most important object defining a $\mathrm{CM}^{2}$. It describes the (Markovian) transition probability of observing the system in $x^{\prime}$, as well as the outcome $z$, given that initially the system was in $x$. With this definition, it follows that

$$
p\left(x^{\prime}, z\right)=\sum_{x} W\left(x^{\prime} z \mid x\right) p(x)
$$

which, classically, is precisely what one would expect from the law of total probability.

Finally, we adapt these ideas to multiple collisions. The initial state of the system is $\rho_{X_{0}}=\sum_{x_{0}} p\left(x_{0}\right)\left|x_{0}\right\rangle\left\langle x_{0}\right|$. The conditional (unnormalized) state after the first collision is obtained by applying Eq. (A10):

$$
\begin{aligned}
\varrho_{X_{1} \mid \zeta_{1}} & =\sum_{x_{1}} p\left(x_{1}, \zeta_{1}\right)\left|x_{1}\right\rangle\left\langle x_{1}\right| \\
p\left(x_{1}, \zeta_{1}\right) & =\sum_{x_{0}} W\left(x_{1} z_{1} \mid x_{0}\right) p\left(x_{0}\right) .
\end{aligned}
$$

Here, recall that $\zeta_{1}=z_{1}$. Similarly, after the second collision, the conditional state will be $\varrho_{X_{2} \mid \zeta_{2}}=$ $\sum_{x_{2}} p\left(x_{2}, \zeta_{2}\right)\left|x_{2}\right\rangle\left\langle x_{2}\right|$, where

$$
p\left(x_{2}, \zeta_{2}\right)=\sum_{x_{0}, x_{1}} W\left(x_{2} z_{2} \mid x_{1}\right) W\left(x_{1} z_{1} \mid x_{0}\right) p\left(x_{0}\right)
$$

Proceeding in this way, we then see that after the $t$ th collision, the state of the conditional system will then be

$$
\varrho_{X_{t} \mid \zeta_{t}}=\sum_{x_{t}} p\left(x_{t}, \zeta_{t}\right)\left|x_{t}\right\rangle\left\langle x_{t}\right|
$$

where

$$
p\left(x_{t}, \zeta_{t}\right)=\sum_{x_{0}, \ldots, x_{t-1}} W\left(x_{t} z_{t-1} \mid x_{t-1}\right) \cdots W\left(x_{1} z_{0} \mid x_{0}\right) p\left(x_{0}\right) .
$$

Tracing over this state and recalling Eq. (9), we then finally obtain the distribution of outcomes

$$
P\left(\zeta_{t}\right)=\sum_{x_{0}, \ldots, x_{t}} W\left(x_{t} z_{t} \mid x_{t-1}\right) \cdots W\left(x_{1} z_{1} \mid x_{0}\right) p\left(x_{0}\right) .
$$

This result is quite important, as it clearly highlights the hidden Markov structure of the present model, discussed in Sec. II.

Summarizing, the incoherent version of a $\mathrm{CM}^{2}$ is completely defined by the transition matrix $W\left(x^{\prime} z \mid x\right)$ in Eq. (A11). This, in turn, depends on the transition matrix $Q\left(x^{\prime} y^{\prime} \mid x y\right)$ in Eq. (A4), which must be unistochastic, and the noise matrix $M\left(z^{\prime} \mid y\right)$, which can be any conditional probability.

\section{APPENDIX B: PROOF OF THE CONDITIONAL VERSION OF THE SECOND LAW}

The proof of Eq. (38) relies on a fundamental inequality of the Holevo information [71]:

$$
I\left(X^{\prime}: z\right) \leq \mathcal{I}\left(X^{\prime}: Y^{\prime}\right) .
$$

It compares the Holevo information for a single collision outcome $z$ with the full quantum mutual information between the system and ancilla, after the collision. This means that, no matter what measurement strategy $\left\{M_{z}\right\}$ one utilizes, the information about the system that can be extracted from the ancilla is at most equal to the full information encoded in the global quantum state $\rho_{X^{\prime} Y^{\prime}}$. This inequality also holds for states conditioned on past outcomes. That is,

$$
G_{t}=I_{c}\left(X_{t}: z_{t} \mid \zeta_{t-1}\right) \leq \mathcal{I}\left(X_{t}: Y_{t}^{\prime} \mid \zeta_{t-1}\right),
$$

where the conditioning is over previous records $\zeta_{t-1}=$ $\left(z_{1}, \ldots, z_{t-1}\right)$ (i.e., those that happened before the present collision) and $G_{t}$ is defined in Eq. (13). This is true since conditional states are still quantum states (provided they are properly normalized), so that Eq. (B1) must still hold.

We now start with Eq. (33) and introduce the splitting (16) to write $\Delta \Sigma_{t}^{c}=\Delta \Sigma_{t}^{u}-G_{t}+L_{t}$. Next we use Eq. (23) for $\Delta \Sigma_{t}^{u}$ and Eq. (13) for $G_{t}$. We then get

$$
\Delta \Sigma_{t}^{c}=\mathcal{I}\left(X_{t}: Y_{t}^{\prime}\right)+D\left(Y_{t}^{\prime} \| Y_{t}\right)-I_{c}\left(X_{t}: z_{t} \mid \zeta_{t-1}\right)+L_{t} .
$$

Using inequality (B2) then shows that

$$
\Delta \Sigma_{t}^{c} \geq \mathcal{I}\left(X_{t}: Y_{t}^{\prime}\right)+D\left(Y_{t}^{\prime}|| Y_{t}\right)-\mathcal{I}\left(X_{t}: Y_{t}^{\prime} \mid \zeta_{t-1}\right)+L_{t} .
$$


Finally, we use Eq. (25) for $\mathcal{I}\left(X_{t}: Y_{t}^{\prime}\right)$. The other mutual information $\mathcal{I}\left(X_{t}: Y_{t}^{\prime} \mid \zeta_{t-1}\right)$ also satisfies a similar formula:

$$
\begin{aligned}
& \mathcal{I}\left(X_{t}: Y_{t}^{\prime} \mid \zeta_{t-1}\right) \\
& \quad=S\left(X_{t} \mid \zeta_{t-1}\right)-S\left(X_{t-1} \mid \zeta_{t-1}\right)+S\left(Y_{t}^{\prime} \mid \zeta_{t-1}\right)-S\left(Y_{t}\right)
\end{aligned}
$$

Thus, the difference between the two mutual information terms can be written as

$$
\begin{aligned}
\mathcal{I}\left(X_{t}: Y_{t}^{\prime}\right)-\mathcal{I}\left(X_{t}: Y_{t}^{\prime} \mid \zeta_{t-1}\right) \\
=\left[S\left(X_{t}\right)-S\left(X_{t} \mid \zeta_{t-1}\right)\right]-\left[S\left(X_{t-1}\right)-S\left(X_{t-1} \mid \zeta_{t-1}\right)\right] \\
\quad+\left[S\left(Y_{t}^{\prime}\right)-S\left(Y_{t}^{\prime} \mid \zeta_{t-1}\right)\right] \\
=-L_{t}+I\left(Y_{t}^{\prime}: \zeta_{t-1}\right),
\end{aligned}
$$

where we recognize, in the first two square brackets, the information loss term $L_{t}$ defined in Eq. (17).

Substituting this back into Eq. (B3) we then finally find Eq. (38). Being a consequence of Eq. (B2), we can also conclude that the first bound in Eq. (38) is saturated by processes where the measurement extracts all the information available. Even in such a limiting case, we still get a nonzero $\Delta \Sigma_{t}^{c}$, so the process is still irreversible.

[1] Kater W. Murch, Kevin L. Moore, Subhadeep Gupta, and Dan M. Stamper-Kurn, Observation of quantummeasurement backaction with an ultracold atomic gas, Nat. Phys. 4, 561 (2008), arXiv:0706.1005v3.

[2] T. P. Purdy, R. W. Peterson, and C. A. Regal, Observation of radiation pressure shot noise on a macroscopic object, Science 339, 801 (2013).

[3] J. Teufel, F. Lecocq, and R. Simmonds, Overwhelming Thermomechanical Motion with Microwave Radiation Pressure Shot Noise, Phys. Rev. Lett. 116, 013602 (2016).

[4] Z. K. Minev, S. O. Mundhada, S. Shankar, P. Reinhold, R. Gutierrez-Jauregui, R. J. Schoelkopf, M. Mirrahimi, H. J. Carmichael, and M. H. Devoret, To catch and reverse a quantum jump mid-flight, Nature 570, 200 (2019), arXiv:1803.00545.

[5] F. Binder, L. A. Correa, C. Gogolin, J. Anders, G Adesso, eds. Thermodynamics in the Quantum Regime - Fundamental Aspects and New Directions (Springer International Publishing, Switzerland, 2019), p. 976.

[6] M. Naghiloo, J. J. Alonso, A. Romito, E. Lutz, and K. W. Murch, Information Gain and Loss for a Quantum Maxwell's Demon, Phys. Rev. Lett. 121, 030604 (2018), arXiv:1802.07205.

[7] Massimiliano Rossi, David Mason, Junxin Chen, and Albert Schliesser, Observing and Verifying the Quantum Trajectory of a Mechanical Resonator, Phys. Rev. Lett. 123, 163601 (2019), arXiv:1812.00928.

[8] Takahiro Sagawa and Masahito Ueda, Second law of Thermodynamics with Discrete Quantum Feedback Control, Phys. Rev. Lett. 100, 080403 (2008), arXiv:0710.0956.
[9] Sosuke Ito and Takahiro Sagawa, Information Thermodynamics on Causal Networks, Phys. Rev. Lett. 111, 180603 (2013), arXiv:1306.2756.

[10] Takahiro Sagawa and Masahito Ueda, Fluctuation Theorem with Information Exchange: Role of Correlations in Stochastic Thermodynamics, Phys. Rev. Lett. 109, 180602 (2012).

[11] Takahiro Sagawa and Masahito Ueda, Role of mutual information in entropy production under information exchanges, New J. Phys. 15, 125012 (2013).

[12] Ken Funo, Yu Watanabe, and Masahito Ueda, Integral quantum fluctuation theorems under measurement and feedback control, Phys. Rev. E 88, 052121 (2013), arXiv:1307.2362.

[13] Cyril Elouard, David A. Herrera-Martí, Maxime Clusel, and Alexia Auffèves, The role of quantum measurement in stochastic thermodynamics, Npj Quant. Inf. 3, 9 (2017), arXiv:1607.02404.

[14] Lorenzo Buffoni, Andrea Solfanelli, Paola Verrucchi, Alessandro Cuccoli, and Michele Campisi, Quantum Measurement Cooling, Phys. Rev. Lett. 122, 070603 (2018), arXiv:1806.07814.

[15] M. Hamed Mohammady and Alessandro Romito, Conditional work statistics of quantum measurements, Quantum 3, 175 (2019), arXiv:1809.09010.

[16] Konstantin Beyer, Kimmo Luoma, and Walter T. Strunz, Work as an external quantum observable and an operational quantum work fluctuation theorem, Phys. Rev. Res. 2, 33508 (2020).

[17] Akira Sone and Sebastian Deffner, Jarzynski equality for conditional stochastic work, arXiv:2010.05835 (2020).

[18] Philipp Strasberg and Andreas Winter, Stochastic thermodynamics with arbitrary interventions, Phys. Rev. E 100, 022135 (2019), arXiv:1905.07990.

[19] Philipp Strasberg, Thermodynamics of quantum causal models: An inclusive, Hamiltonian approach, Quantum 4, 240 (2020), 1911.01730.

[20] Alessio Belenchia, Luca Mancino, Gabriel T. Landi, and Mauro Paternostro, Entropy production in continuously measured quantum systems, Npj Quant. Inf. 6, 97 (2020), arXiv:1908.09382.

[21] Shoichi Toyabe, Takahiro Sagawa, Masahito Ueda, Eiro Muneyuki, and Masaki Sano, Experimental demonstration of information-to-energy conversion and validation of the generalized jarzynski equality, Nat. Phys. 6, 988 (2010), arXiv:1009.5287.

[22] J. V. Koski, V. F. Maisi, J. P. Pekola, and D. V. Averin, Experimental realization of a szilard engine with a single electron, Proc. Natl. Acad. Sci. U.S.A. 111, 13786 (2014), arXiv:1402.5907.

[23] N Cottet, S Jezouin, L Bretheau, P. Campagne-Ibarcq, Q Ficheux, Janet Anders, Alexia Auffèves, R. Azouit, P. Rouchon, and B. Huard, Observing a quantum Maxwell demon at work, Proc. Natl. Acad. Sci. U.S.A 114, 7561 (2017), arXiv:1702.05161.

[24] Maxime Debiossac, David Grass, Jose Joaquin Alonso, Eric Lutz, and Nikolai Kiesel, Thermodynamics of continuous non-markovian feedback control, Nat. Commun. 11, 1360 (2020), arXiv:1904.04889.

[25] H. M. Wiseman and G. J. Milburn, Quantum Measurement and Control (Cambridge University Press, New York, 2009). 
[26] Kurt Jacobs, Quantum Measurement Theory and its Applications (Cambridge University Press, Cambridge, 2014).

[27] Massimiliano Rossi, Luca Mancino, Gabriel T. Landi, Mauro Paternostro, Albert Schliesser, and Alessio Belenchia, Experimental Assessment of Entropy Production in a Continuously Measured Mechanical Resonator, Phys. Rev. Lett. 125, 080601 (2020), arXiv:2005.03429.

[28] Gabriel T. Landi and Mauro Paternostro, Irreversible entropy production, from quantum to classical, arXiv:2009. 07668.

[29] Amikam Levy and Ronnie Kosloff, The local approach to quantum transport may violate the second law of thermodynamics, EPL (Europhysics Letters) 107, 20004 (2014), arXiv: 1402.3825 .

[30] G. De Chiara, G. Landi, A. Hewgill, B. Reid, A. Ferraro, A. J. Roncaglia, and M. Antezza, Reconciliation of quantum local master equations with thermodynamics, New J. Phys. 20, 113024 (2018), arXiv:1808.10450.

[31] Jayaseetha Rau, Relaxation phenomena in spin and harmonic oscillator systems, Phys. Rev. 129, 1880 (1963).

[32] Valerio Scarani, Mário Ziman, Peter Štelmachovič, Nicolas Gisin, Vladimír Bužek, and Vladimír Bužek, Thermalizing Quantum Machines: Dissipation and Entanglement, Phys. Rev. Lett. 88, 097905 (2002), arXiv:0110088 [quant-ph].

[33] M. Ziman, P. Štelmachovič, V. Buzžek, M. Hillery, V. Scarani, and N. Gisin, Diluting quantum information: An analysis of information transfer in system-reservoir interactions, Phys. Rev. A 65, 042105 (2002).

[34] Berthold-Georg Englert and Giovanna Morigi, in Coherent Evolution in Noisy Environments - Lecture Notes in Physics, edited by A. Buchleitner and K. Hornberger (Springer, Berlin, Heidelberg, 2002), p. 611, arXiv:0206116 [quant-ph].

[35] Stéphane Attal and Yan Pautrat, From repeated to continuous quantum interactions, Annales Henri Poincaré 7, 59 (2006), arXiv:0311002 [math-ph].

[36] D. Karevski and T. Platini, Quantum Nonequilibrium Steady States Induced by Repeated Interactions, Phys. Rev. Lett. 102, 207207 (2009), arXiv:0904.3527.

[37] C Pellegrini and F Petruccione, Non-markovian quantum repeated interactions and measurements, J. Phys. A 42, 425304 (2009), arXiv:0903.3859.

[38] V. Giovannetti and G. M. Palma, Master Equations for Correlated Quantum Channels, Phys. Rev. Lett. 108, 040401 (2012).

[39] Tomáš Rybár, Sergey N. Filippov, Mário Ziman, and Vladimír Bužek, Simulation of indivisible qubit channels in collision models, J. Phys. B 45, 154006 (2012), arXiv: 1202.6315 .

[40] Philipp Strasberg, Gernot Schaller, Tobias Brandes, and Massimiliano Esposito, Quantum and Information Thermodynamics: A Unifying Framework Based on Repeated Interactions, Phys. Rev. X 7, 021003 (2017), arXiv:1610.01829.

[41] Franklin L. S. Rodrigues, Gabriele De Chiara, Mauro Paternostro, and Gabriel T. Landi, Thermodynamics of Weakly Coherent Collisional Models, Phys. Rev. Lett. 123, 140601 (2019), arXiv:1906.08203.

[42] Felipe Barra, The thermodynamic cost of driving quantum systems by their boundaries, Sci. Rep. 5, 14873 (2015), arXiv:1509.04223.
[43] Emmanuel Pereira, Heat, work, and energy currents in the boundary-driven XXZ spin chain, Phys. Rev. E 97, 022115 (2018).

[44] Francesco Ciccarello, Collision models in quantum optics, Quantum Measurements Quantum Metrology 4, 53 (2017), arXiv:1712.04994.

[45] Jonathan A. Gross, Carlton M. Caves, Gerard J. Milburn, and Joshua Combes, Qubit models of weak continuous measurements: Markovian conditional and opensystem dynamics, Quantum Sci. Technol. 3, 024005 (2018), arXiv: 1710.09523.

[46] The code can be downloaded https://www.wolframcloud. com/obj/gtlandi/Published/CM2_qubit_models.nbhere.

[47] Physically speaking, the unitary $U_{t}$ is generated by a physical Hamiltonian that acts for a certain time $\tau$ between $X$ and $Y$. The time steps $t=0,1,2, \ldots$ are thus in units of $\tau$. Since we are interested only in the state after each step $\tau$, we do not need to specify the full interaction Hamiltonian, only its net effect, which is summarized by the operator $U$.

[48] A Darwiche, Modeling and Reasoning with Bayesian Networks (Cambridge University Press, Cambridge, 2009).

[49] R. E. Neapolitan, Learning Bayesian Networks (PrenticeHall, Upper Saddle River, 2003).

[50] A. S. Holevo, Bounds for the quantity of information transmitted by a quantum communication channel, Problems Inf. Transmission 9, 177 (1973).

[51] M. A. Nielsen and I. L. Chuang, Quantum Computation and Quantum Information (Cambridge University Press, Cambridge, 2000).

[52] Q. Ficheux, S. Jezouin, Z. Leghtas, and B. Huard, Dynamics of a qubit while simultaneously monitoring its relaxation and dephasing, Nat. Commun. 9, 1 (2018), arXiv: 1711.01208 .

[53] Enrico Fermi, Thermodynamics (Dover Publications Inc., New York, 1956), p. 160.

[54] Massimiliano Esposito, Katja Lindenberg, and Christian Van den Broeck, Entropy production as correlation between system and reservoir, New J. Phys. 12, 013013 (2010), arXiv:0908.1125.

[55] Gonzalo Manzano, Jordan M. Horowitz, and Juan M. R. Parrondo, Quantum Fluctuation Theorems for Arbitrary Environments: Adiabatic and Non-Adiabatic Entropy Production, Phys. Rev. X 8, 031037 (2018), arXiv:1710. 00054.

[56] Heinz Peter Breuer, Quantum jumps and entropy production, Phys. Rev. A 68, 032105 (2003), arXiv:0306047 [quant-ph].

[57] Jordan M. Horowitz, Quantum-trajectory approach to the stochastic thermodynamics of a forced harmonic oscillator, Phys. Rev. E 85, 031110 (2012).

[58] G. T. Landi, M. Paternostro, and A. Belenchia, Informational steady-states and conditional entropy production in continuously monitored systems: the continuous-variable scenario, (2021), to appear.

[59] P. Z. Peebles, Probability, Random Variables, and Random Signal Principles (McGraw-Hill, Singapore, 1993), 3rd ed., p. 448.

[60] G. Chiribella, G. M. D'Ariano, and P. Perinotti, Quantum Circuit Architecture, Phys. Rev. Lett. 101, 060401 (2008), arXiv:0712.1325. 
[61] Felix A. Pollock, César Rodríguez-Rosario, Thomas Frauenheim, Mauro Paternostro, and Kavan Modi, Nonmarkovian quantum processes: Complete framework and efficient characterization, Phys. Rev. A 97, 012127 (2018), arXiv:1512.00589.

[62] Felix A. Pollock, César Rodríguez-Rosario, Thomas Frauenheim, Mauro Paternostro, and Kavan Modi, Operational Markov Condition for Quantum Processes, Phys. Rev. Lett. 120, 040405 (2018), arXiv:1801.09811.

[63] Philipp Strasberg, Operational approach to quantum stochastic thermodynamics, Phys. Rev. E 100, 022127 (2019), arXiv:1810.00698.

[64] Jose Joaquin Alonso, Eric Lutz, and Alessandro Romito, Thermodynamics of Weakly Measured Quantum Systems, Phys. Rev. Lett. 116, 080403 (2016), arXiv:1508. 00438 .

[65] M. Naghiloo, D. Tan, P. M. Harrington, J. J. Alonso, E. Lutz, A. Romito, and K. W. Murch, Heat and Work Along Individual Trajectories of a Quantum Bit, Phys. Rev. Lett. 124, 110604 (2020), arXiv:1703.05885.
[66] H. J. Groenewold, A problem of information gain by quantal measurements, Int. J. Theor. Phys. 4, 327 (1971).

[67] Masanao Ozawa, On information gain by quantum measurements of continuous observables, J. Math. Phys. 27, 759 (1986).

[68] Bartłomiej Gardas and Sebastian Deffner, Quantum fluctuation theorem for error diagnostics in quantum annealers, Sci. Rep. 8, 1 (2018).

[69] Lorenzo Buffoni and Michele Campisi, Thermodynamics of a quantum annealer, Quantum Sci. Technol. 5, 035013 (2020).

[70] Valeria Cimini, Stefano Gherardini, Marco Barbieri, Ilaria Gianani, Marco Sbroscia, Lorenzo Buffoni, Mauro Paternostro, and Filippo Caruso, Experimental characterization of the energetics of quantum logic gates, Npj Quantum Inf. 6, 1 (2020).

[71] Harold Ollivier and Wojciech Hubert Zurek, Quantum Discord: A Measure of the Quantumness of Correlations, Phys. Rev. Lett. 88, 017901 (2001), arXiv:0105072 [quant-ph]. 